American Journal of Pharmacology and Toxicology 2 (4): 146-147, 2007

ISSN 1557-4962

(C) 2007 Science Publications

\title{
Anomalies in a Fetus Exposed to Methotrexate in the First Trimester
}

\author{
Landon B. Lorenz and Eric J. Knudtson \\ Department of Obstetrics and Gynecology, Section of Maternal Fetal Medicine \\ University of Oklahoma Health Sciences Center, Oklahoma City, Oklahoma, USA
}

\begin{abstract}
The increasing use of methotrexate for multiple indications in reproductive age women will likely lead to more frequent incidence of fetal exposure. Methotrexate is sometimes used for medical termination of first trimester pregnancy in combination with misoprostol. Teratogenesis in women exposed to low-dose methotrexate used for treatment of rheumatic conditions has been reported, but it has also been suggested that there is no teratogenic risk associated with low-dose exposure. Because medical termination with methotrexate and misoprostol has high success rates, reports of anomalies in ongoing pregnancies are rare. A 19-year-old gravida 3, para 1 presented in the third trimester after failing an attempt at medical termination with methotrexate. Ultrasonography revealed an absent right kidney and concern for stenosis of the tricuspid valve. After delivery, the absence of the right kidney was confirmed as well as a ventricular septal defect. Low-dose methotrexate, even in a single dose, may be associated with fetal anomalies. Caution must be taken with the use of methotrexate for termination of pregnancy. Patient reliability, adequate follow-up and appropriate counseling for the risk of failure are essential.
\end{abstract}

Key words: Medical termination, abortion, teratogenesis

\section{INTRODUCTION}

Methotrexate is a folic acid antagonist with a variety of clinical uses. Reproductive age women are commonly treated with methotrexate for neoplastic processes, psoriasis, ulcerative colitis, lupus and rheumatoid arthritis. Usually used in combination with misoprostol, methotrexate is also used for medical termination of pregnancy prior to 8 weeks gestation. Typically, $50 \mathrm{mg} \mathrm{m} \mathrm{m}^{2}$ is administered intramuscularly followed 3-7 days later by oral or vaginal administration of $800 \mathrm{mg}$ of misoprostol. When given to women at less than 8 weeks gestation, failure rates are generally reported as approximately $10 \%$; however, failure rates as low as $2 \%$ have been seen some studies $^{[1-3]}$. In cases of failure, abortion is normally completed by surgical evacuation.

The antineoplastic and anti-inflammatory effects of folic acid antagonists are secondary to the inhibition of dihydrofolate reductase. This disrupts the synthesis of DNA, RNA and protein precursors. Because of this mechanism of action, methotrexate preferentially targets rapidly proliferating cell lines; consequently, it is potentially harmful to the developing fetus.

Cases of fetal anomalies associated with methotrexate have been reported. Overall, there is a paucity of reports. Aminopterin (methotrexate is a methyl derivative of aminopterin) exposures and subsequent malformations are more widely reported. Amionopterin syndrome is a constellation of central nervous system and skeletal abnormalities described in the literature which includes cranial dysostosis, hyperteloreism, anomalies of the external ears, micrognathia and posterior cleft palate. Dextrocardia has also been reported with aminopterin exposure ${ }^{[4]}$.

Most women with exposure to methotrexate and ongoing pregnancies have normal outcomes. It has been suggested by some studies that there is no strong teratogenic risk when methotrexate is used in low-doses and is discontinued as soon as pregnancy is discovered $^{[5]}$. Higher doses are typically used for termination of pregnancy, but there is little data regarding outcomes of failed terminations involving methotrexate.

Case: A 19-year-old gravida 3, para 1-0-1-1 underwent an attempted medical termination at an outside facility at approximately 6 weeks gestation. Obstetrical history was significant for a prior medical termination at 9 weeks gestation and a term cesarean delivery. She was given a single intramuscular injection of $50 \mathrm{mg} \mathrm{m}$ methotrexate. It was unclear whether subsequent administration of misoprostol was part of the treatment plan, or if the patient failed to follow-up. The patient

Corresponding Author: Landon Lorenz, P.O. Box 26901, WP 2410, Oklahoma City, Oklahoma 73190, (405) 271-8470 
denies any subsequent bleeding or passage of tissue. Approximately 6 months later, the patient presented to the OB/GYN residents' clinic of this hospital with no interval prenatal care. She was diagnosed with and treated for trichomonas vaginalis at the initial prenatal visit.

Initial ultrasonography revealed a 31 week fetus, 3 vessel cord, amniotic fluid index of $7.2 \mathrm{~cm}$, an abnormal appearing tricuspid valve, absent right kidney and the left kidney was multicystic in appearance. The tricuspid valve was echogenic and stenotic-appearing. Four weeks later, the amniotic fluid volume was normal but the tricuspid valve and left kidney remained abnormal. The pregnancy progressed to term without complication.

At 39 weeks, the patient presented to the hospital in active labor and underwent a repeat cesarean section without complication. The female liveborn infant weighed $3590 \mathrm{~g}$ at birth with Apgar scores of 8 and 9. An echocardiogram revealed a small to medium-sized ventriculoseptal defect. An abdominal sonogram was normal except for absence of the right kidney and mild hydronephrosis of the left kidney. Ultrasound encephalography was normal and at 25 days-of-age a voiding cystourethrogram was obtained and showed no vesicoureteral reflux. Follow-up left renal sonograms were obtained at 2 and 6 months-of-age and were essentially normal.

Of note, the patient re-presented on postoperative day 12 with postpartum eclampsia. She was discharged after a short hospitalization.

\section{DISCUSSION}

Methotrexate has become increasingly popular in the treatment of non-neoplastic conditions, increasing the likelihood of fetal exposure. Reliable contraception is vital in reproductive age women undergoing treatment with methotrexate for medical conditions. Additionally, when methotrexate is used for medical termination of pregnancy, the risk of failure needs to be discussed with the patient. As with mifepristone, this regimen needs to be administered by providers and patients who understand the risks and are comfortable with performing surgical dilatation and curettage as backup.

Reported malformations in fetuses exposed to folic acid antagonists have largely been skeletal or craniofacial in nature. This case suggests that the possibility of a larger range of anomalies cannot be excluded. An Ovid MEDLINE search of the literature published since 1950 was performed with keywords "methotrexate" and "teratogenesis," "abnormalities," “teratogens," or "congenital heart defect." Only eight reported cases of fetal anomalies were identified where methotrexate exposure was related to the failure of a medical termination of pregnancy. In most of these cases, defects were craniofacial and/or skeletal in nature. None involved abnormalities of the urinary system $^{[6-10]}$.

In summary, our case demonstrates that failed medical termination of pregnancy with methothrexate is associated with a wider range of fetal anomalies than previously reported. Patients should be adequately counseled as to the risk of treatment failure. As with mifepristone and misoprostol, administration should be limited to clinicians and patients who understand the risks. Additionally, providers must be willing and able to perform a surgical dilatation and curettage in cases of treatment failure.

\section{REFERENCES}

1. Morton A. Stenchever, 2001. Comprehensive Gynecology. Mosby, 13.

2. Goldberg, A, M. Greenberg and P. Darney, 2001. Misoprostol and pregnancy. N. Engl. J. Med., 344: 38-47.

3. Powell, H. and H. Eckert, 1971. Methotrexateinduced congenital malformations. Med. J. Aust., 2: 1076-1077.

4. Buckley, L., C. Bullaboy, L. Leichtman and M. Marquez, 1997. Multiple congenital anomalies associated with weekly low-dose methotrexate treatment of the mother. Arthr. Rheumat., 40: 971-973.

5. Lewden, B., T. Vial, E. Elefant, A. Nelva, P. Carlier and J. Descotes, 2004. Low dose methotrexate in the first trimester of pregnancy: Results of a french collaborative study. J. Rheumatol., 31: 2360-2365.

6. Goffman, D., D.S. Cole, P. Bobby and D.J. Garry, 2006. Failed methotrexate termination of pregnancy: A case report. J. Perinatol., 26: 645-7.

7. Yedlinsky, N.T., F.C. Morgan and P.W. Whitecar, 2005. Anomalies associated with failed methotrexate and misoprostol termination. Obstetr. Gynecol., 105: 1203-5.

8. Adam, M.P., M.A. Manning, A.E. Beck, A. Kwan, G.M. Enns, C. Clericuzio and H.E. Hoyme, 2003. Am. J. Med. Genet., 123: 72-8.

9. Wheeler, M., P. O’Meara and M. Stanford, 2002. Fetal methotrexate and misoprostol exposure: The past revisited. Teratology, 66: 73-6.

10. Bawle, E.V., J.V. Conard and L. Weiss, 1998. Adult and two children with fetal methotrexate syndrome. Teratology, 57: 51-5. 